\title{
Managing Concerning Behaviors in Patients Prescribed Opioids for Chronic Pain: A Delphi Study
}

\author{
Jessica S. Merlin, MD, MBA ${ }^{1,2}$, Sarah R. Young, $M D, M B A^{1,3}$, Joanna L. Starrels, $M D, M B A^{4}$, \\ Soraya Azari, $M D, M B A^{5}$, E. Jennifer Edelman, $M D, M B A^{6}$, Jamie Pomeranz, $M D, M B A^{7}$, \\ Payel Roy, MD, $M B A^{8}$, Shalini Saini, MD, MBA 9 , William C. Becker, $M D, M B A^{6,10}$, and \\ Jane M. Liebschutz, MD, MBA ${ }^{8}$
}

\begin{abstract}
'Division of Infectious Diseases, University of Alabama at Birmingham, Birmingham, AL, USA; ${ }^{2}$ Division of Gerontology, Geriatrics, and Palliative Care, University of Alabama at Birmingham, Birmingham, AL, USA; ${ }^{3}$ Department of Social Work, College of Community and Public Affairs, Binghamton University, Binghamton, NY, USA; ${ }^{2}$ Division of General Internal Medicine, Albert Einstein College of Medicine and Montefiore Medical Center, Bronx, NY, USA; 5 Division of General Internal Medicine, San Francisco General Hospital, University of California San Francisco, San Francisco, CA, USA; ${ }^{6}$ Department of Internal Medicine, Yale University School of Medicine, New Haven, CT, USA; ${ }^{7}$ Department of Occupational Therapy, University of Florida, Gainesville, FL, USA; ${ }^{8}$ Clinical Addiction Research and Education (CARE) Unit, Section of General Internal Medicine, Boston Medical Center, Boston University School of Medicine, Boston, MA, USA; ${ }^{9}$ Department of Medicine, Information Technology, University of Alabama at Birmingham, Birmingham, AL, USA; ${ }^{10}$ VA Connecticut Healthcare System, West Haven, CT, USA.
\end{abstract}

BACKGROUND: Current guideline-recommended monitoring of patients prescribed long-term opioid therapy (LTOT) for chronic pain will likely result in increased identification of behaviors of concern for misuse and addiction, but there is a dearth of empiric evidence about how these behaviors should be managed.

OBJECTIVE: To establish expert consensus about treatment approaches for common and challenging concerning behaviors that arise among patients on LTOT. DESIGN: We used a Delphi approach, which allows for generation of consensus.

PARTICIPANTS: Participants were clinical experts in chronic pain and opioid prescribing recruited from professional societies and other expert groups.

MAIN MEASURES: The Delphi process was conducted online, and consisted of an initial brainstorming round to identify common and challenging behaviors, a second round to identify management strategies for each behavior, and two rounds to establish consensus and explore disagreement/uncertainty.

KEY RESULTS: Forty-two participants completed round 1, 22 completed round 2, 30 completed round 3, and 28 completed round 4 . Half of round 1 participants were female $(52 \%)$, and the majority were white $(83 \%)$. Most (71\%) were physicians, and most participants practiced in academic primary (40\%) or specialty care (19\%).The most frequently cited common and challenging behaviors were missing appointments, taking opioids for symptoms other than pain, using more opioid medication than prescribed, asking for an increase in opioid dose, aggressive behavior, and alcohol and other substance use. Across behaviors, participants agreed that patient education

Electronic supplementary material The online version of this article (https://doi.org/10.1007/s11606-017-4211-y) contains supplementary material, which is available to authorized users.

Received April 28, 2017

Revised September 12, 2017

Accepted October 25, 2017

Published online December 4, 2017 and information gathering were important approaches. Participants also agreed that stopping opioids is not important initially, but if initial approaches do not work, tapering opioids and stopping opioids immediately may become important approaches.

CONCLUSIONS: This study presents clinical expert consensus on how to manage concerning behaviors among patients on LTOT. Future research is needed to investigate how implementing these management strategies would impact patient outcomes, practice and policy.

J Gen Intern Med 33(2):166-76

DOI: $10.1007 /$ s11606-017-4211-y

(c) Society of General Internal Medicine 2017

\section{INTRODUCTION}

Opioid overdose mortality in the United States has more than quadrupled since 2000, and in 2015 more than 33,000 Americans died of an overdose involving opioids. ${ }^{1}$ Opioid use disorder and non-fatal overdose have also increased markedly. ${ }^{1,2}$ From 1991 to 2013, the number of prescriptions of opioid medications nearly tripled. ${ }^{3}$ Primary care providers, who provide the majority of chronic pain care in the US, are on the front lines of this crisis and have an important role in ensuring that the opioids they prescribe are used as safely as possible.

The 2016 Centers for Disease Control and Prevention (CDC) Guideline for Prescribing Opioids for Chronic Pain was addressed to primary care providers. It recommended judicious prescribing of opioids for chronic pain and routine monitoring of patients on long-term opioid therapy (LTOT), using strategies such as urine drug testing and reviewing prescription drug monitoring program data. ${ }^{4}$ These strategies aim to identify and address problems and avoid serious harms. ${ }^{5,6}$

However, when following the CDC guideline in treating patients prescribed LTOT for chronic pain, clinicians encounter patient behaviors that raise concerns about misuse, substance 
use disorders, or increased risk for overdose. We call these behaviors "concerning behaviors"; other terms include "aberrant drug-related behaviors" and "opioid misuse." They include behaviors such as illicit drug use or overuse of prescribed opioids, which are associated with increased risk of serious harms. ${ }^{8}$ Such behaviors are common; a recent systematic review found that rates of opioid misuse averaged 21-29\% across diverse settings. ${ }^{9}$ Concerning behaviors may signal an important underlying problem such as opioid or other substance use disorder. ${ }^{10}$ Opioid prescribers, who are often primary care providers, lack training in pain and addiction, and find assessment and management of these behaviors to be challenging. ${ }^{11-14}$

The CDC guideline provides useful general recommendations for clinicians to reassess the role of LTOT for chronic pain. However, advice on addressing specific concerning behaviors is beyond the scope of the guideline. Therefore, many important clinical questions remain unaddressed. ${ }^{15}$ For example, how should a clinician respond to cocaine use, or to aggressive behavior? When is it necessary to taper or stop opioids? Our authorship team has substantial clinical and research expertise in this field but has not found any empiric evidence to address these questions. Given the lack of empiric data, a Delphi approach can address these gaps in evidence through generation of consensus by a panel of experts. ${ }^{16,17}$ Therefore, we conducted a Delphi study, the objective of which was to establish consensus treatment approaches for the most important concerning behaviors that arise among patients on LTOT.

\section{METHODS}

We previously published a detailed description of the methods for this four-round Delphi study conducted from March 2015 to August 2016. ${ }^{17}$ Here, we summarize our approach and focus on the consensus-generating rounds (rounds 3 and 4).

\section{Participants}

We recruited participants from four groups: American Academy of Pain Medicine members who identified anesthesia, internal medicine, neurology, physical medicine and rehabilitation, or psychiatry as their specialty; members of the Society of General Internal Medicine's Pain Medicine and Alcohol, Tobacco, and Other Drug Use Interest Groups; Department of Veterans Affairs Veterans Health Administration (VA) pain experts, known as "Pain Points of Contact"; and Safe and Competent Opioid Prescribing Education (SCOPE) of Pain ${ }^{18}$ trainers. We emailed potential participants with an invitation to take part in the study, conducted online using Qualtrics survey software (2015; Provo, UT). Individuals were eligible to participate if they provided direct patient care to adults with chronic pain on LTOT in an ambulatory setting and if they identified opioid prescribing for chronic pain as an area of expertise. We made the a priori decision to randomly choose one participant per round to receive a $\$ 100$ online retailer gift card to maximize participation. The entire Delphi process occurred online via Qualtrics.

\section{Rounds}

The Delphi process consisted of four rounds. Participants were not asked to complete any "homework" between rounds, but rather completed each round in "real time."

Round 1: To identify the most clinically relevant behaviors, we asked participants to list all behaviors and other concerning signs they considered to be problematic among patients on LTOT. Next, they selected the two most common and two most challenging behaviors from their list. Using a thematic approach, we grouped the behaviors into similar categories (e.g., "early refill requests" and "running out of medication early" into "using more opioid medication than prescribed"). ${ }^{19,20}$ We tabulated the common and challenging behaviors to identify those most frequently cited, and focus here on the six behaviors most frequently identified as challenging, common or both.

Round 2: Round 1 participants were eligible for round 2. We asked participants how they would typically manage each of the most important behaviors from round 1 . We analyzed these responses using a thematic approach. Management strategies that were part of usual clinical care (e.g., determine differential diagnosis) or appeared across all behaviors were not analyzed further. We identified the six most common management strategies for each behavior to further examine in round 3.

Round 3: Round 1 participants were eligible to participate in round 3 . We presented participants with the common and challenging concerning behaviors identified in round 1 and the corresponding management strategies identified in round 2 . Participants were asked to rate the importance of each management strategy on a scale of 1-9 $(1=$ not at all important, $9=$ extremely important). Free-text boxes allowed participants to clarify their responses or describe additional information they felt was necessary to evaluate the management strategy's importance ("clarifying information").

Disagreement was defined a priori as one-third or more participant responses in the "not important" range AND one-third in the "important" range for a particular strategy. We considered the absence of disagreement to be consensus, and used the median rating to categorize importance. We considered responses $\leq 3$ as "not important," $4-6$ as "uncertain," and $\geq 7$ as "important." We reviewed the clarifying information to identify potential "branch points" that could lead to different management strategies. We also looked for instances in 
which the free-text responses suggested that the behavior needed further clarification.

Round 4: All participants who completed round 3 were eligible to participate in round 4 . The purpose of round 4 was threefold. First, if the behavior needed further clarification, we revised the behavior and presented participants with the same management strategies as in round 3. Second, to achieve consensus where there was disagreement, we presented the requested clarifying information, the participants' round 3 scores, and the group's median score, and gave participants the opportunity to adjust their scores. Third, we sought to determine the importance of management strategies with median scores of 4 6 (uncertain importance) in round 3 . We presented participants with clinical scenarios that differed based on the potential branch points identified in round 3, and asked them to assume that all strategies that attained consensus as "important" had already been implemented (see Appendix). Participants rated the importance of the management strategy, and the same procedures as in round 3 were used to determine consensus. These procedures led to additional questions in round 4 to those in round 3 ; in one case, clarifying a behavior resulted in breaking one behavior into two behaviors.

Each round remained open for 3 weeks, and weekly reminder emails were sent. Thus, the number of participants in each round represents the participants who responded to the survey within that period of time.

Based on participants' ratings, we present consensus findings for each of the six most common and most challenging concerning behaviors. Management strategies that achieved consensus as "important" or "not important" are described as such. Management strategies where there was either disagreement or consensus as to uncertain importance are described as of "uncertain importance." Findings related to marijuana use in the context of LTOT will be considered in a separate publication, given the heterogeneity of participants' prescribing environments.

\section{Ethical Approval}

This study was approved by the institutional review board of the University of Alabama at Birmingham (UAB).

\section{RESULTS}

Forty-two participants completed round 1. Of those, 33 (79\%) participants completed round 2, and $30(71 \%)$ completed round 3. Of those who completed round 3, 28 (93\%) completed round 4.

Half of round 1 participants were female (52\%), and the majority were white (83\%). Most (71\%) were physicians, of whom $90 \%$ completed residencies in internal medicine. Most participants practiced in academic primary (40\%) or specialty care (19\%); $48 \%$ practiced in VA Medical Centers. All regions of the US were represented (Northeast: $31 \%$, Midwest: 14\%, South: $31 \%$, West: $24 \%$ ).

Based on participants' responses to round 1 and our qualitative analysis, the most frequently identified common and challenging behaviors were (1) missing appointments, (2) taking opioids for symptoms other than pain, (3) using more opioid medication than prescribed, (4) asking for an increase in opioid dose, (5) aggressive behavior, and (6) alcohol and other substance use including cocaine, methamphetamine, benzodiazepines and heroin (Table 1). Management strategies that were part of usual care or were uniform across behaviors included assessing risk and safety concerns, identifying pain symptoms, considering the differential diagnosis of the behavior, discussing the pros and cons of the behavior with the patient, and providing patient education.

Of the 73 management strategies presented to participants in round 3, 50 attained consensus as "important," 19 attained consensus as being of "uncertain importance," and 3 attained consensus as "not important." There was disagreement for one management strategy - stopping opioids in response to cocaine use. For a summary of these results, see Appendix Table 2.

Of the 75 management strategies presented to participants in round 4, 26 attained consensus as "important," 29 attained consensus as being of "uncertain importance," and 14 attained consensus as "not important." There was disagreement on five management strategies. For a summary of these results, see Appendix Table 3.

Here, we provide a brief description of management strategies for each behavior.

\section{Behavior 1: Missing appointments}

Participants achieved consensus that the following initial management strategies are important: determine whether a pattern of behavior has been present, review opioid treatment agreement with the patient, require appointment attendance if opioids are to be continued, and give patient at least one chance to change their behavior. Participants also achieved consensus that stopping opioids immediately is not important. Participants agreed that if a patient continues to miss appointments, tapering opioids is important, while stopping opioids immediately without providing additional prescriptions is of uncertain importance.

Behavior 2: Taking opioids for symptoms other than pain (e.g., for anxiety, depression, sleep, or to produce euphoria 
Table 1 Most Frequently Identified Common and Challenging Concerning Behaviors in Patients on Long-Term Opioid Therapy*

\begin{tabular}{|c|c|c|c|}
\hline$\overline{\text { Behavior }}$ & Examples & Common $^{\dagger}$ & Challenging $^{\dagger}$ \\
\hline Behavior 1: Missing appointments & & 3 & 6 \\
\hline $\begin{array}{l}\text { Behavior 2: Taking opioids for symptoms other } \\
\text { than pain }\end{array}$ & Anxiety, depression, sleep, or to produce euphoria & 5 & 5 \\
\hline $\begin{array}{l}\text { Behavior 3: Using more opioid medication than } \\
\text { prescribed }\end{array}$ & $\begin{array}{l}\text { Unsanctioned dose escalation, early refill requests, running out of } \\
\text { medication early }\end{array}$ & 22 & 7 \\
\hline $\begin{array}{l}\text { Behavior 4: Asking for an increase in opioid } \\
\text { dose }\end{array}$ & $\begin{array}{l}\text { Demanding, repeatedly asking, or asking in the absence of a clinical } \\
\text { change in pain }\end{array}$ & 3 & 6 \\
\hline $\begin{array}{l}\text { Behavior 5: Aggressive behavior towards } \\
\text { provider or staff }\end{array}$ & $\begin{array}{l}\text { Outbursts of anger, rude or demanding behavior, threats towards } \\
\text { staff }\end{array}$ & 2 & 9 \\
\hline Behavior 6: Alcohol and other substance use & Methamphetamine, cocaine, benzodiazepines, heroin, and marijuana & 6 & 3 \\
\hline
\end{tabular}

*Values given are the number of participants who selected the behavior as one of the two most common or two most challenging concerning behaviors $\dagger N=42$ participants in round 1

Participants achieved consensus that the following initial management strategies are important: discuss or refer for nonopioid therapies, and make a referral, e.g., to a psychologist, psychiatrist, or to an addiction treatment program. Participants also achieved consensus that stopping opioids immediately is not important. Participants agreed that if the behavior persists, tapering opioids is important, while stopping opioids immediately without providing additional prescriptions is of uncertain importance.

Behavior 3: Using more opioid medication than prescribed (e.g., unsanctioned dose escalation, early refill requests, running out of medication early)

Participants achieved consensus that the following initial management strategies are important: review opioid treatment agreement with the patient, order urine toxicology tests that day and more frequently, provide prescriptions at shorter intervals, discuss or refer for non-opioid therapies, discuss or assess for an opioid use disorder, and determine whether a pattern of behavior has been present. Participants also achieved consensus that stopping opioids immediately is not important. Participants agreed that if a pattern of concerning behavior is present, denying early refills is important, and if the patient has an opioid use disorder, participants achieved consensus that the following strategies are important: utilize pill counts, refer to addiction treatment or related services, refer to a pain specialist, and taper opioids.

Behavior 4: Asking for an increase in opioid dose (i.e., demanding or repeatedly asking)

Participants achieved consensus that the following initial management strategies are important: discuss or refer for nonopioid therapies and refer to a pain specialist. Participants agreed that increasing the opioid dose was of uncertain importance in patients taking $\leq 100$ morphine milligram equivalents (MME) per day, and that increasing the dose is not important in patients taking >100 MME per day.

Behavior 5: Aggressive behavior towards provider or staff
In rounds 3 and 4 , comments clarified that participants viewed this behavior as a combination of two behaviors: verbally aggressive behavior where there is no concern for provider or staff safety (e.g., outbursts of anger, rude or demanding behavior towards providers or staff), and aggressive behavior where there is concern for provider or staff safety (e.g., threats towards staff). Therefore, in round 4, these two behaviors were separated. In both cases, participants achieved consensus that the following initial management strategies are important: ask for third party to be present, listen to patient concerns, let patient know that their behavior will not be tolerated, and determine whether a pattern of behavior has been present. When there is no concern for provider or staff safety, participant consensus was that calling security and tapering opioids are of uncertain importance, and stopping opioids immediately and discharging the patient from the practice are not important. If there is a concern for provider or staff safety, participants agreed that calling security, tapering opioids, stopping opioids immediately, and discharging the patient are important strategies.

Behavior 6: Substance use

Participants achieved consensus that several initial strategies are important across all substances: discuss or assess for a substance use disorder, refer for addiction treatment or related services, review opioid treatment agreement with the patient, order urine toxicology tests more frequently, and determine whether a pattern of behavior has been present. After these initial strategies, consensus differed for alcohol and other substances.

\section{Alcohol Use}

Based on round 4 responses, participants agreed that tapering or stopping opioids is not important if no pattern of at-risk alcohol use is present. However, if there is a pattern of at-risk alcohol use but not an alcohol use disorder, participants achieved consensus that tapering opioids is of uncertain importance and that stopping opioids immediately is not important. If a pattern of at-risk alcohol use and an alcohol use 
disorder are present, participants achieved consensus that tapering opioids is important, and that stopping opioids immediately is of uncertain importance.

\section{Other Substance Use}

In round 3, there was disagreement about whether to stop opioids in response to cocaine use. Round 3 comments underscored the importance of knowing the urine drug test results. In round 4, participants achieved consensus that stopping opioids immediately is important with cocaine use if there is concern that the patient is not taking the opioids (e.g., the urine drug screen has been repeatedly negative for the prescribed opioid).

Participants achieved consensus that referring for pharmacotherapy for opioid use disorder, stopping opioid therapy immediately, and tapering opioids is important if any heroin use is present. For cocaine, methamphetamine, and benzodiazepines, if a pattern of substance use with or without a substance use disorder is present, participants agreed that tapering opioids is important, and that stopping opioid therapy immediately is of uncertain importance. If there is no pattern of repeated use present and no substance use disorder, participants agreed that tapering opioids is of uncertain importance.

\section{DISCUSSION}

This study provides the first consensus-level evidence to frontline clinicians about the importance of various strategies for addressing common and challenging concerning behaviors among patients prescribed LTOT for chronic pain. The study's strengths include the use of a methodologically rigorous Delphi approach. ${ }^{16,21}$ Consensus was achieved on most management strategies.

We note that across all behaviors, which often co-occur in clinical practice, common themes arose. For most behaviors, management approaches involved first having additional discussions with the patient and determining whether a pattern of behavior was present, and only then making major decisions (e.g., tapering or stopping opioids). Notably, tapering or stopping opioids were never considered as a first step, but rather as something to contemplate depending on how the patient responded to other initial steps. Reviewing the opioid treatment agreement, which can be seen as a proxy for reviewing the plan and the risks and benefits of opioids with the patient, also featured prominently. All of this implies a systematic, patient-centered process involving frequent clinic visits, open communication, observation of how the patient responds, and a flexible management plan. The authors are aware that this may be in contrast to contemporary clinical practice, in which providers may feel compelled to make major decisions abruptly (e.g., taper or discontinue opioids) due to state or local policies or licensure concerns, regardless of whether these decisions are warranted. ${ }^{22}$

Our findings underscore challenges inherent in managing concerning behaviors in primary care. First, a recurring theme in our findings is referral for non-opioid pharmacologic and non-pharmacologic therapies. These could include cognitive behavioral therapy, ${ }^{23}$ pain self-management programs, ${ }^{24}$ and complementary treatments such as acupuncture. ${ }^{25}$ Despite their known efficacy, access to such approaches is often limited due to scarcity and lack of insurance coverage. ${ }^{26}$ The 2016 Department of Health and Human Services National Pain Strategy and CDC Guideline for Prescribing Opioids for Chronic Pain call for greater availability of such therapies, but no clear plan exists for expanding access on a national scale. ${ }^{4,27}$ Until this happens, providers and patients may continue to struggle with limited treatment options for chronic pain beyond LTOT.

We also found that the presence of a substance use disorder is a key branch point in decision-making. However, primary care providers often lack training in diagnosing and treating substance use disorders, ${ }^{13}$ and access to addiction specialty services is limited. ${ }^{28}$ Therefore, our findings highlight the well-established need to enhance primary providers' training in substance use disorder diagnosis and treatment and to expand the addiction workforce. ${ }^{29}$ We also note that the approaches generated by this Delphi process do not include early referral to addiction specialists for many behaviors. There are several possible explanations for this finding. With the possible exception of heroin use, none of these behaviors in and of themselves represents addiction absent additional evaluation, and so a referral to an addiction specialist may have seemed premature. For example, a referral to an addiction specialist for missing appointments, or even for escalating opioid use, may not be appropriate. Furthermore, the scarcity of addiction specialty services may lead primary care providers to refer only more clear-cut cases of active addiction, managing others on their own.

This study has limitations. A major limitation of the Delphi method is that it relies on opinions, in this case provider opinions about clinical care, which can be flawed. Also, the management strategies are based on how participants reported they would manage concerning behaviors, but have not been tested in clinical practice. Though there is an urgent need for information about when to use which management strategies, ${ }^{15}$ further research will be important in evaluating the impact of the strategies on patient- and provider-level outcomes, and if they are effective, in refining their content, developing setting-specific adaptations and support, exploring novel methods of delivery, and providing specific guidance as to how providers should implement these strategies (e.g., develop algorithms). If providers choose to implement these strategies, we advise they be used to as an adjunct to sound clinical judgment.

In sum, these findings are an important first step in a new program of research that could provide clinical guidance in 
managing concerning behaviors among patients on LTOT for chronic pain. Given the current opioid prescribing and addiction epidemic, and the recent attention to the importance of opioid risk mitigation, management of newly uncovered concerning behaviors will become an even more important part of caring for patients on LTOT. Future research should investigate the impact of these management strategies on patients and providers.

Acknowledgements: The authors wish to acknowledge the following study participants, who completed all four Delphi rounds and have given permission for us to publish their names (alphabetically):

Osvaldo Acosta-Rodriguez

J. Mark Bailey

Geetanjali Chander

Phoebe Cushman

Mark Eisenberg

Janette Elliott

Joseph Frank

Shannon Gully

Jared Klein

Erin Krebs

Marc LaRochelle

Hilary Moshe.

Daniel Pomerantz

Ilene Robeck

Daniel Tobin

Corresponding Author: Jessica S. Merlin, MD, MBA; Division of Infectious Diseases, University of Alabama at Birmingham, Birmingham, AL, USA (e-mail: merlinjs@pitt.edu).

Funding Three authors were supported by NIH career development awards awarded by the National Institute of Mental Health (K23MH104073 [JSM]) and the National Institute on Drug Abuse (K23DA027719 [JLS] and K12DA033312 [EJE]).

\section{Compliance with Ethical Standards:}

Conflict of Interest: JLS receives research support from the Opioid Post-Marketing Requirements Consortium, via subcontract with the Clinical Directors Network. The remaining authors declare that they have no conflict of interest.

\section{REFERENCES}

1. Rudd RA, Aleshire N, Zibbell JE, Gladden RM. Increases in Drug and Opioid Overdose Deaths - United States, 2000-2014. MMWR Morb Mortal Wkly Rep. 2016;64(50-51):1378-82.

2. Saha TD, Kerridge BT, Goldstein RB, Chou SP, Zhang H, Jung J, et al. Nonmedical Prescription Opioid Use and DSM-5 Nonmedical Prescription Opioid Use Disorder in the United States. J Clin Psychiatry. 2016;77(6):772-80

3. IMS's National Prescription Audit (NPA) \& Vector One @: National (VONA).

4. Dowell D, Haegerich TM, Chou R. CDC Guideline for Prescribing Opioids for Chronic Pain - United States, 2016. MMWR Recomm Rep. 2016;65(1):1-49.

5. Weiner SG, Griggs CA, Mitchell PM, Langlois BK, Friedman FD, Moore RL, et al. Clinician Impression Versus Prescription Drug Monitoring Program Criteria in the Assessment of Drug-Seeking Behavior in the Emergency Department. Ann Emerg Med. 2013.

6. Starrels JL, Becker WC, Alford DP, Kapoor A, Williams AR, Turner BJ. Systematic review: treatment agreements and urine drug testing to reduce opioid misuse in patients with chronic pain. Ann Intern Med. 2010;152(11):712-20

7. Colburn JL, Jasinski DR, Rastegar DA. Long-term opioid therapy, aberrant behaviors, and substance misuse: comparison of patients treated by resident and attending physicians in a general medical clinic. J Opioid Manag. 2012;8(3):153-60.

8. Meltzer EC, Rybin D, Meshesha LZ, Saitz R, Samet JH, Rubens SL, et al. Aberrant Drug-Related Behaviors: Unsystematic Documentation Does Not Identify Prescription Drug Use Disorder. Pain Med. 2012.

9. Vowles KE, McEntee ML, Julnes PS, Frohe T, Ney JP, van der Goes DN. Rates of opioid misuse, abuse, and addiction in chronic pain: a systematic review and data synthesis. Pain. 2015;156(4):569-76.

10. Alford DP. Chronic back pain with possible prescription opioid misuse. JAMA. 2013;309(9):919-25.

11. Dobscha SK, Corson K, Flores JA, Tansill EC, Gerrity MS. Veterans affairs primary care clinicians' attitudes toward chronic pain and correlates of opioid prescribing rates. Pain Med. 2008;9(5):564-71.

12. Merlin JS, Turan JM, Herbey I, Westfall AO, Starrels JL, Kertesz SG, et al. Aberrant drug-related behaviors: a qualitative analysis of medical record documentation in patients referred to an HIV/chronic pain clinic. Pain Med. 2014;15(10):1724-33.

13. Jamison RN, Sheehan KA, Scanlan E, Matthews M, Ross EL. Beliefs and attitudes about opioid prescribing and chronic pain management: survey of primary care providers. J Opioid Manag. 2014;10(6):375-82.

14. Lum PJ, Little S, Botsko M, Hersh D, Thawley RE, Egan JE, et al. Opioid-prescribing practices and provider confidence recognizing opioid analgesic abuse in HIV primary care settings. J Acquir Immune Defic Syndr. 2011;56 Suppl 1:S91-7.

15. Chou R, Ballantyne JC, Fanciullo GJ, Fine PG, Miaskowski C. Research gaps on use of opioids for chronic noncancer pain: findings from a review of the evidence for an American Pain Society and American Academy of Pain Medicine clinical practice guideline. J Pain. 2009;10(2):147-59.

16. Keeney S, Hasson F, McKenna HP. The Delphi technique in nursing and health research. Chichester: Wiley-Blackwell; 2011.

17. Merlin JS, Young SR, Azari S, Becker WC, Liebschutz JM, Pomeranz $\mathbf{J}$, et al. Management of problematic behaviours among individuals on long-term opioid therapy: protocol for a Delphi study. BMJ Open. 2016;6(5):e011619.

18. Alford DP, Zisblatt L, Ng P, Hayes SM, Peloquin S, Hardesty I, et al. SCOPE of Pain: An Evaluation of an Opioid Risk Evaluation and Mitigation Strategy Continuing Education Program. Pain Med. 2015.

19. Braun V, Clarke V. Using thematic analysis in psychology. Qual Res Psychol. 2006;3(2)77-101. Available from: http://eprints.uwe.ac.uk/ 11735.

20. Attride-Stirling $\mathbf{J}$. Thematic networks: an analytic tool for qualitative research. Qual Res. 1(3), 385-405.; 2001.

21. Diamond IR, Grant RC, Feldman BM, Pencharz PB, Ling SC, Moore AM, et al. Defining consensus: a systematic review recommends methodologic criteria for reporting of Delphi studies. J Clin Epidemiol. 2014;67(4):401-9.

22. Kertesz SG. Turning the Tide or Riptide? the Changing Opioid Epidemic. Subst Abus. 2017;38(1):3-8

23. Kerns RD, Sellinger J, Goodin BR. Psychological treatment of chronic pain. Annu Rev Clin Psychol. 2011;7:411-34.

24. Kroenke K, Bair MJ, Damush TM, Wu J, Hoke S, Sutherland J, et al Optimized antidepressant therapy and pain self-management in primary care patients with depression and musculoskeletal pain: a randomized controlled trial. JAMA. 2009;301(20):2099-110.

25. Vickers AJ, Cronin AM, Maschino AC, Lewith G, Macpherson H, Foster NE, et al. Acupuncture for Chronic Pain: Individual Patient Data Meta-analysis. Arch Intern Med. 2012:1-10.

26. Cheatle MD. Facing the challenge of pain management and opioid misuse, abuse and opioid-related fatalities. Expert Rev Clin Pharmacol. 2016;9(6):751-4.

27. Department of Health and Human Services UG. National Pain Strategy: A comprehensive population health strategy for pain. Accessed on July 6 , 2016 at: https://iprcc.nih.gov/docs/HHSNational_Pain_Strategy.pdf.

28. U.S. Department of Health and Human Services (HHS), Office of the Surgeon General. Facing Addiction in America: The Surgeon General's Report on Alcohol, Drugs, and Health. Washington, DC: HHS; November 2016.

29. SAMSHA. http://www.samhsa.gov/medication-assisted-treatment/ qualify-nps-pas-waivers. 


\section{APPENDIX}

Table 2 Delphi Round 3 Results: Management Strategies for the Most Common and Challenging Concerning Behaviors in Patients on LongTerm Opioid Therapy

\begin{tabular}{l}
\hline Behavior \\
\hline Strategies found to be "important" (recommend) \\
Behavior 1: Missing appointment
\end{tabular}

Behavior 1: Missing appointment

Behavior 2: Taking opioids for symptoms other than pain (e.g., anxiety, depression, sleep, or to produce euphoria)

Behavior 3: Using more opioid medication than prescribed (e.g., unsanctioned dose escalation, early refill requests, running out of medication early)

Behavior 4: Asking for increase in opioid dose (e.g., demanding, repeatedly asking, or asking in the absence of a clinical change in pain)

Behavior 5: Aggressive behavior towards provider or staff (e.g., outbursts of anger, rude or demanding behavior, threats towards staff)

Behavior 6: Substance use-alcohol

Behavior 6: Substance use - methamphetamine

Behavior 6: Substance use - cocaine

Behavior 6: Substance use - benzodiazepine

Behavior 6: Substance use-heroin

Strategies found to be of "uncertain" importance (consider)

Behavior 1: Missing appointments

Behavior 2: Taking opioids for symptoms other than pain (e.g., anxiety, depression, sleep, or to produce euphoria)
Management strategy

Median

Determine whether a pattern of behavior has been present (e.g., by talking to the patient or reviewing records) Review opioid treatment agreement with the patient Require appointment attendance if opioids are to be continued

Give patient at least one chance to change behavior

Discuss or refer for non-opioid therapies (non-opioid pharmacologic therapies, non-opioid non-pharmacologic therapies)

Make a referral (e.g., to a psychologist, psychiatrist, or to an addiction treatment program)

Determine whether pattern of behavior is present

Review opioid treatment agreement

Order urine toxicology test that day

Order urine toxicology tests more frequently

Provide prescriptions in shorter intervals

Discuss or refer for non-opioid therapies (e.g., non-opioid

pharmacologic therapies, non-opioid non-pharmacologic

therapies)

Discuss or assess for a substance use disorder

Individualize my response to the patient's behavior

Avoid dose escalation

Discuss or refer for non-opioid therapies (e.g., non-opioid

pharmacologic therapies, non-opioid non-pharmacologic therapies)

Make a referral to a pain specialist

Listen to patient's concerns

Determine whether a pattern of behavior has been present

(e.g., by talking to the patient or reviewing records)

Ask for third party to be present (e.g., clinic manager, nurse, social worker)

Let patient know that behavior will not be tolerated

Determine whether a pattern of behavior has been present

(e.g., by talking to the patient or reviewing records)

Discuss or assess for a substance use disorder

Refer to addiction treatment or related services

Review opioid treatment agreement with the patient

Order urine toxicology tests more frequently

Determine whether a pattern of behavior has been present

(e.g., by talking to the patient or reviewing records)

Discuss or assess for a substance use disorder

Refer to addiction treatment or related services

Review opioid treatment agreement with the patient

Order urine toxicology tests more frequently

Taper opioids

Determine whether a pattern of behavior has been present

(e.g., by talking to the patient or reviewing records)

Discuss or assess for a substance use disorder

Refer to addiction treatment or related services

Review opioid treatment agreement with the patient

Order urine toxicology tests more frequently

Determine whether a pattern of behavior has been present

(e.g., by talking to the patient or reviewing records)

Discuss or assess for a substance use disorder

Refer to addiction treatment or related services

Review opioid treatment agreement with the patient

Order urine toxicology tests more frequently

Determine whether a pattern of behavior has been present

(e.g., by talking to the patient or reviewing records)

Discuss or assess for a substance use disorder

Consider pharmacotherapy for opioid use disorder

(buprenorphine, methadone)

Refer to addiction treatment or related services

Review opioid treatment agreement with the patient

Order urine toxicology tests more frequently

Taper opioids 
Table 2. (continued)

\begin{tabular}{|c|c|c|}
\hline Behavior & Management strategy & Median \\
\hline \multirow{5}{*}{$\begin{array}{l}\text { Behavior 3: Using more opioid medication than prescribed (e.g., } \\
\text { unsanctioned dose escalation, early refill requests, running out of } \\
\text { medication early) }\end{array}$} & Utilize pill counts & 6 \\
\hline & Make a referral to addiction treatment & 5.5 \\
\hline & Make a referral to a pain specialist & 5 \\
\hline & Deny early refill request, even on first ask & 5 \\
\hline & Taper opioids & 5 \\
\hline \multirow{2}{*}{$\begin{array}{l}\text { Behavior 4: Asking for increase in opioid dose (e.g., demanding, } \\
\text { repeatedly asking, or asking in the absence of a clinical change in pain) }\end{array}$} & $\begin{array}{l}\text { Increase dose if reasonable (including time-limited trial of } \\
\text { dose increase) }\end{array}$ & 5 \\
\hline & Make a referral to addiction treatment services & 5 \\
\hline \multirow{4}{*}{$\begin{array}{l}\text { Behavior 5: Aggressive behavior towards provider or staff (e.g., outbursts } \\
\text { of anger, rude or demanding behavior, threats towards staff) }\end{array}$} & Call security & 6 \\
\hline & Taper opioids & 6 \\
\hline & Stop opioids immediately (no additional prescriptions) & 5 \\
\hline & Discharge patient from the practice & 5 \\
\hline \multirow{2}{*}{ Behavior 6: Substance use - alcohol } & Taper opioids & 6.5 \\
\hline & $\begin{array}{l}\text { Alcohol: Stop opioid therapy immediately (no additional } \\
\text { prescriptions }\end{array}$ & 6.5 \\
\hline Behavior 6: Substance use-methamphetamine & Stop opioid therapy immediately (no additional prescriptions) & 5 \\
\hline Behavior 6: Substance use - cocaine & Taper opioids & 6 \\
\hline \multirow[t]{2}{*}{ Behavior 6: Substance use-benzodiazepine } & Taper opioids & 6 \\
\hline & Stop opioid therapy immediately (no additional prescriptions) & 5 \\
\hline \multirow[t]{2}{*}{ Behavior 6: Substance use-alcohol } & Taper opioids & 6.5 \\
\hline & $\begin{array}{l}\text { Alcohol: Stop opioid therapy immediately (no additional } \\
\text { prescriptions }\end{array}$ & 6.5 \\
\hline \multicolumn{3}{|l|}{ Strategies found to be "not important" (not recommended) } \\
\hline Behavior 1: Missing appointments & Stop opioid therapy immediately (no additional prescriptions) & 3 \\
\hline $\begin{array}{l}\text { Behavior 2: Taking opioids for symptoms other than pain (e.g., anxiety, } \\
\text { depression, sleep, or to produce euphoria) }\end{array}$ & Stop opioids immediately (no additional prescriptions) & 3 \\
\hline $\begin{array}{l}\text { Behavior 3: Using more opioid medication than prescribed (e.g., } \\
\text { unsanctioned dose escalation, early refill requests, running out of } \\
\text { medication early) }\end{array}$ & Stop opioids immediately (no additional prescriptions) & 3 \\
\hline \multicolumn{3}{|l|}{ Strategies for which there was disagreement } \\
\hline Behavior 6: Substance use - cocaine & $\begin{array}{l}\text { Cocaine: Stop opioid therapy immediately (no additional } \\
\text { prescriptions) }\end{array}$ & N/A \\
\hline
\end{tabular}

Table 3 Delphi Round 4 Results: Management Strategies for the Most Common and Challenging Concerning Behaviors in Patients on LongTerm Opioid Therapy

\begin{tabular}{|c|c|c|c|c|}
\hline Scenario & Case & Strategy & $\begin{array}{l}\text { Consensus or } \\
\text { disagreement? }\end{array}$ & Median \\
\hline $\begin{array}{l}\text { Behavior 1: Missing appointments } \\
\text { You are seeing a patient in clinic with } \\
\text { the following behavior: Missing } \\
\text { appointments. In the previous round, } \\
\text { participants agreed that the following } \\
\text { management strategies are an important } \\
\text { response to this behavior: } \\
\text { - Determine whether a pattern of behavior } \\
\text { has been present (e.g., by talking to the } \\
\text { patient or reviewing records). } \\
\text { - Review opioid treatment agreement with } \\
\text { the patient } \\
\text { - Require appointment attendance if } \\
\text { opioids are to be continued } \\
\text { - Give patient at least one chance to } \\
\text { change behavior } \\
\text { Now imagine that you have implemented } \\
\text { all of the above strategies while } \\
\text { continuing to prescribe opioids. Please } \\
\text { consider the following case: }\end{array}$ & $\begin{array}{l}\text { Case 1. You continue to use these } \\
\text { strategies for a reasonable period of } \\
\text { time and the patient continues to miss } \\
\text { appointments. Given this information: }\end{array}$ & Taper opioids & $\mathrm{C}$ & 7 \\
\hline
\end{tabular}


Table 3. (continued)

\begin{tabular}{|c|c|c|c|c|}
\hline Scenario & Case & Strategy & $\begin{array}{l}\text { Consensus or } \\
\text { disagreement? }\end{array}$ & Median \\
\hline $\begin{array}{l}\text { Behavior 2: Taking opioids for symptoms } \\
\text { other than pain (e.g., anxiety, depression, } \\
\text { sleep, or to produce euphoria) } \\
\text { You are seeing a patient in clinic with the } \\
\text { following behavior: Taking opioids for } \\
\text { symptoms other than pain (e.g., for } \\
\text { anxiety, depression, sleep, or to produce } \\
\text { euphoria). In the previous round, } \\
\text { participants agreed that the following } \\
\text { management strategies are an important } \\
\text { response to this behavior: } \\
\text { - Discuss or refer for non-opioid therapies } \\
\text { (e.g., non-opioid pharmacologic therapies, } \\
\text { non-opioid non-pharmacologic therapies) } \\
\text { - Make a referral (e.g., to a psychologist, } \\
\text { psychiatrist, or to addiction treatment } \\
\text { program) } \\
\text { Now please imagine that you have } \\
\text { implemented these strategies. Please } \\
\text { consider the following case: }\end{array}$ & $\begin{array}{l}\text { Case } 1 \text {. You continue to use the } \\
\text { above strategies for a reasonable } \\
\text { period of time and the patient } \\
\text { continues to take opioids for } \\
\text { symptoms other than pain. Given this } \\
\text { information: }\end{array}$ & $\begin{array}{l}\text { Taper opioids } \\
\text { Stop opioid therapy } \\
\text { immediately (no additional } \\
\text { prescriptions) }\end{array}$ & $\begin{array}{l}\mathrm{C} \\
\mathrm{C}\end{array}$ & $\begin{array}{l}8 \\
4\end{array}$ \\
\hline Behavior 3: Using more opioid & Case 1. You assess the patient. You & Utilize pill counts & $\mathrm{C}$ & 5 \\
\hline $\begin{array}{l}\text { medication than prescribed (e.g., } \\
\text { unsanctioned dose escalation, early refill }\end{array}$ & $\begin{array}{l}\text { determine that a pattern of concerning } \\
\text { behavior HAS NOT been present, }\end{array}$ & $\begin{array}{l}\text { Make a referral to addiction } \\
\text { treatment }\end{array}$ & $\mathrm{C}$ & 2 \\
\hline $\begin{array}{l}\text { requests, running out of medication early) } \\
\text { You are seeing a patient in clinic with the }\end{array}$ & $\begin{array}{l}\text { and there is NO CLEAR BASIS for a } \\
\text { diagnosis of opioid use disorder. }\end{array}$ & $\begin{array}{l}\text { Make a referral to a pain } \\
\text { specialist }\end{array}$ & $\mathrm{C}$ & 6 \\
\hline $\begin{array}{l}\text { following behavior: Using more opioid } \\
\text { medication than prescribed (e.g., }\end{array}$ & Given this information: & $\begin{array}{l}\text { Deny early refill, even on first } \\
\text { ask }\end{array}$ & $\mathrm{C}$ & 6 \\
\hline unsanctioned dose escalation, early & & Taper opioids & $\mathrm{C}$ & 4 \\
\hline $\begin{array}{l}\text { refill requests, running out of } \\
\text { medication early). In the previous round, }\end{array}$ & $\begin{array}{l}\text { Case 2. You assess the patient. You } \\
\text { determine that a pattern of concerning }\end{array}$ & $\begin{array}{l}\text { Utilize pill counts } \\
\text { Make a referral to addiction }\end{array}$ & $\begin{array}{l}\mathrm{C} \\
\mathrm{C}\end{array}$ & $\begin{array}{l}6 \\
5\end{array}$ \\
\hline $\begin{array}{l}\text { management strategies are an important } \\
\text { response to this behavior: }\end{array}$ & $\begin{array}{l}\text { is NO CLEAR BASIS for a diagnosis } \\
\text { of opioid use disorder. Given this }\end{array}$ & $\begin{array}{l}\text { Make a referral to a pain } \\
\text { specialist }\end{array}$ & $\mathrm{C}$ & 6 \\
\hline $\begin{array}{l}\text { - Determine whether a pattern of behavior } \\
\text { has been present (e.g., by talking to the } \\
\text { patient or reviewing records) }\end{array}$ & information: & $\begin{array}{l}\text { Deny early refill, even on first } \\
\text { ask } \\
\text { Taner onioids }\end{array}$ & $\mathrm{C}$ & 8 \\
\hline $\begin{array}{l}\text { patient or reviewing records) } \\
\text { - Review opioid treatment agreement with }\end{array}$ & Case 3. You assess the patient. You & $\begin{array}{l}\text { Iaper opiolds } \\
\text { Utilize pill counts }\end{array}$ & $\mathrm{C}$ & $\begin{array}{l}5 \\
7\end{array}$ \\
\hline $\begin{array}{l}\text { the patient. } \\
\text { - Order urine toxicology tests that day }\end{array}$ & & $\begin{array}{l}\text { Make a referral to addiction } \\
\text { treatment }\end{array}$ & $\mathrm{C}$ & 9 \\
\hline $\begin{array}{l}\text { - Order urine toxicology tests more } \\
\text { frequently }\end{array}$ & $\begin{array}{l}\text { YOU STRONGLY SUSPECT a } \\
\text { diagnosis of opioid use disorder. }\end{array}$ & $\begin{array}{l}\text { Make a referral to a pain } \\
\text { specialist }\end{array}$ & $\mathrm{C}$ & 7 \\
\hline $\begin{array}{l}\text { - Provide prescriptions at shorter intervals } \\
\text { (e.g., 2-week supply) }\end{array}$ & Given this information: & $\begin{array}{l}\text { Deny early refill, even on first } \\
\text { ask }\end{array}$ & $\mathrm{C}$ & 9 \\
\hline $\begin{array}{l}\text { Discuss or refer for non-opioid therapies } \\
\text { (non-opioid pharmacologic therapies, } \\
\text { non-opioid non-pharmacologic therapies) } \\
\text { - Discuss or assess for a substance use } \\
\text { disorder } \\
\text { - Individualize my response to the } \\
\text { patient's behavior } \\
\text { Now imagine that you have implemented } \\
\text { all of these strategies. Please consider the } \\
\text { following cases: }\end{array}$ & & Taper opioids & $\mathrm{C}$ & 7 \\
\hline $\begin{array}{l}\text { Behavior 4: Asking for increase in opioid } \\
\text { dose (e.g., demanding, repeatedly asking, } \\
\text { or asking in the absence of a clinical }\end{array}$ & $\begin{array}{l}\text { Case } 1 . \text { The patient is on a dose of } \\
\text { opioids equivalent to less than } 50 \mathrm{mg} \\
\text { of morphine per day. }\end{array}$ & $\begin{array}{l}\text { Increase dose if reasonable } \\
\text { (including time-limited trial of } \\
\text { dose increase) }\end{array}$ & $\mathrm{C}$ & 5 \\
\hline $\begin{array}{l}\text { change in pain) } \\
\text { You are seeing a patient in clinic with the } \\
\text { following behavior: Asking for increase }\end{array}$ & $\begin{array}{l}\text { Case 2: The patient is on a dose of } \\
\text { opioids equivalent to } 50-100 \mathrm{mg} \text { of } \\
\text { morphine per day. }\end{array}$ & $\begin{array}{l}\text { Increase dose if reasonable } \\
\text { (including time-limited trial of } \\
\text { dose increase) }\end{array}$ & $\mathrm{C}$ & 4.5 \\
\hline $\begin{array}{l}\text { in opioid dose (e.g., demanding or } \\
\text { repeatedly asking). In the previous } \\
\text { round, participants agreed that the } \\
\text { following management strategies are an } \\
\text { important response to this behavior: } \\
\text { - Discuss or refer for non-opioid therapies } \\
\text { (e.g., non-opioid pharmacologic therapies, } \\
\text { non-opioid non-pharmacologic therapies) } \\
\text { - Make a referral to a pain specialist } \\
\text { Now imagine that you have implemented } \\
\text { all of the above strategies. Please consider } \\
\text { the following cases: }\end{array}$ & $\begin{array}{l}\text { Case } 3 \text {. The patient is on a dose of } \\
\text { opioids equivalent to greater than } 100 \\
\text { mg of morphine per day. }\end{array}$ & $\begin{array}{l}\text { Increase dose if reasonable } \\
\text { (including time-limited trial of } \\
\text { dose increase) }\end{array}$ & $\mathrm{C}$ & 2 \\
\hline
\end{tabular}


Table 3. (continued)

\begin{tabular}{l}
\hline Scenario \\
\hline Behavior 5 (now divided into two \\
behaviors): Verbally aggressive behavior \\
where there is no concern for provider or \\
staff safety (e.g., outbursts of anger, rude \\
or demanding behavior towards providers \\
or staff)
\end{tabular}

Behavior 5 (now divided into two behaviors): Aggressive behavior where there is concern for provider or staff safety (e.g., threats towards staff)
Behavior 6: Substance use - cocaine Cocaine use with urine drug screen repeatedly negative for prescribed opioids Behavior 6: Substance use - alcohol You are seeing a patient in clinic with the following behavior: Alcohol use. In the previous round, participants agreed that the following management strategies are an important response to this behavior: - Determine whether a pattern of behavior has been present (e.g., by talking to the patient or reviewing records)

- Discuss or assess for a substance use disorder

- Refer for addiction treatment or related services

- Review opioid treatment agreement with the patient

- Order urine toxicology tests more frequently

Now imagine that you have implemented all of these strategies. Please consider the following cases:

\section{N/A}

N/A

Case 1. You assess the patient. You determine that a pattern of at risk alcohol use HAS NOT been present, and there is NO CLEAR BASIS for a diagnosis of alcohol use disorder. Given this information:

Case 2. You assess the patient. You determine that a pattern of at risk alcohol use HAS been present, but there is NO CLEAR BASIS for a diagnosis of alcohol use disorder. Given this information:

Case 3. You assess the patient. You determine that a pattern of at risk alcohol use HAS been present, and YOU STRONGLY SUSPECT a diagnosis of alcohol use disorder. Given this information:
Strategy

Consensus or

Median

disagreement?

Listen to patient's concerns

Determine whether a pattern of

C

8

behavior has been present (e.g.

by talking to the patient or

reviewing records)

Ask for third party to be present (e.g., clinic manager, nurse, social worker)

Call security

Taper opioids

Stop opioids immediately (no

additional prescriptions)

Let patient know that their

behavior will not be tolerated

Discharge patient from the

practice

Listen to patient's concerns

Determine whether a pattern of

behavior has been present (e.g.

by talking to the patient or

reviewing records)

Ask for third party to be

present (e.g., clinic manager,

nurse, social worker)

Call security

Taper opioids

Stop opioids immediately (no additional prescriptions)

Let patient know that their

behavior will not be tolerated

Discharge patient from the practice

Stop therapy immediately

8

Taper opioids

Stop opioid therapy

immediately (no additional

prescriptions)

Taper opioids

Stop opioid therapy

immediately (no additional

prescriptions)

Switch patient to

buprenorphine/naloxone if you

have a waiver to prescribe this medication, or refer patient to someone who does

Taper opioids

Stop opioid therapy

immediately (no additional

prescriptions)
C

C

C

C

C

C

C

5

C

$\mathrm{C}$

9

C

9

C

C

(n)

5 .5

9

9

C

C

6

C

C 8

D N/A


Table 3. (continued)

\begin{tabular}{l}
\hline \hline Scenario \\
\hline Behavior 6: Substance \\
use - methamphetamine \\
You are seeing a patient in clinic with the \\
following behavior: Methamphetamine \\
use. In the previous round, participants \\
agreed that the following management \\
strategies are an important response to this \\
behavior: \\
- Determine whether a pattern of behavior \\
has been present (e.g., by talking to the \\
patient or reviewing records) \\
- Discuss or assess for a substance use \\
disorder \\
- Refer for addiction treatment or related \\
services \\
- Review opioid treatment agreement with \\
the patient
\end{tabular}

the patient

- Order urine toxicology tests more

frequently

Now imagine that you have implemented all of the above strategies. Please consider the following cases:

Behavior 6: Substance use - cocaine You are seeing a patient in clinic with the following behavior: Cocaine use. In the previous round, participants agreed that the following management strategies are an important response to this behavior:

- Determine whether a pattern of behavior has been present (e.g., by talking to the patient or reviewing records)

- Discuss or assess for a substance use disorder

- Refer for addiction treatment or related services

- Review opioid treatment agreement with the patient

- Order urine toxicology tests more frequently

Now imagine that you have implemented all of the above strategies. Please consider the following cases:

Behavior 6: Substance

use-benzodiazepine

You are seeing a patient in clinic with the following behavior: Benzodiazepine use (illicit use or non-medical use of prescription benzodiazepine). In the previous round, participants agreed that the following management strategies are an important response to this behavior:

- Determine whether a pattern of behavior has been present (e.g., by talking to the patient or reviewing records)

- Discuss or assess for a substance use disorder

- Refer for addiction treatment or related services

- Review opioid treatment agreement with the patient

- Order urine toxicology tests more frequently

Now imagine that you have implemented all of the above strategies. Please consider the following cases:

Case Strategy

\section{Strategy}

Consensus or

Median

Case 1. You assess the patient. You determine that a pattern of repeated methamphetamine use HAS NOT been present, and there is NO CLEAR BASIS for a diagnosis of methamphetamine use disorder.

Given this information:

Case 2. You assess the patient. You determine that a pattern of repeated methamphetamine use HAS been present, but there is NO CLEAR BASIS for a diagnosis of methamphetamine use disorder. Given this information:

Case 3. You assess the patient. You determine that a pattern of repeated methamphetamine use HAS been present, and YOU STRONGLY

SUSPECT a diagnosis of methamphetamine use disorder. Given this information:

Case 1. You assess the patient. You determine that a pattern of repeated cocaine use HAS NOT been present, and there is NO CLEAR BASIS for a diagnosis of cocaine use disorder. Given this information: Please rate the importance of the following management strategy:

Case 2. You assess the patient. You determine that a pattern of repeated cocaine use HAS been present but there is NO CLEAR BASIS for a diagnosis of cocaine use disorder. Given this information:

Case 3. You assess the patient. You determine that a pattern of repeated cocaine use HAS been present, and YOU STRONGLY SUSPECT a diagnosis of cocaine use disorder. Given this information:

Case 1. You assess the patient. You determine that a pattern of repeated benzodiazepine use HAS NOT been present, and there is NO CLEAR BASIS for a diagnosis of benzodiazepine use disorder. Given this information:

Case 2. You assess the patient. You determine that a pattern of repeated benzodiazepine use HAS been present, but there is NO CLEAR BASIS for a diagnosis of benzodiazepine use disorder. Given this information:

Case 3. You assess the patient. You determine that a pattern of repeated benzodiazepine use HAS been present, and YOU STRONGLY SUSPECT a diagnosis of benzodiazepine use disorder. Given this information:
Taper opioids

Switch patient to

buprenorphine/naloxone if you have a waiver to prescribe this medication, or refer patient to someone who does

Stop opioid therapy

Taper opioids

Switch patient to

buprenorphine/naloxone if you have a waiver to prescribe this medication, or refer patient to someone who does

Stop opioid therapy

immediately (no additional

prescriptions)

Taper opioids

Switch patient to

buprenorphine/naloxone if you have a waiver to prescribe this medication, or refer patient to

someone who does

Stop opioid therapy

immediately (no additional

prescriptions)

Taper opioids

Switch patient to

disagreement?

$\begin{array}{ll}\mathrm{C} & 5 \\ \mathrm{C} & 3.5\end{array}$

buprenorphine/naloxone if you

have a waiver to prescribe this medication, or refer patient to someone who does

Stop opioid therapy

Taper opioids

Switch patient to

buprenorphine/naloxone if you have a waiver to prescribe this medication, or refer patient to someone who does

Stop opioid therapy

Taper opioids

Switch patient to

buprenorphine/naloxone if you have a waiver to prescribe this medication, or refer patient to someone who does

Stop opioid therapy

Taper opioids

Switch patient to

buprenorphine/naloxone if you

have a waiver to prescribe this medication, or refer patient to someone who does

Stop opioid therapy

Taper opioids

Switch patient to

buprenorphine/naloxone if you have a waiver to prescribe this medication, or refer patient to someone who does

Stop opioid therapy

Taper opioids

Switch patient to

buprenorphine/naloxone if you have a waiver to prescribe this medication, or refer patient to someone who does

Stop opioid therapy
$\mathrm{C}$
$\mathrm{C}$
$\mathrm{C}$ 2.5 